OECD: Are Students Ready for a Technology-Rich World? What PISA Studies Tell Us OECD, 2006, 138 pp.

ISBN: 9264036083

\title{
Are Students Ready for a Technology-Rich World?
}

\section{Laura Naves Arnaldos ${ }^{101}$}

Las tecnologías de la información y la comunicación juegan un papel fundamental en el desarrollo de las modernas economías y sociedades, permitiendo un intercambio de ideas, capital e información atravesando fronteras y con mínimos costes.

Invertir en estas tecnologías repercute en la economía global. Desde los años 80 se ha podido comprobar que los países que forman parte de la OCDE y que invierten en tecnologías de la información han incrementado, en relación directa con esta inversión, su PIB.

Además, el uso de estas tecnologías tiene profundas implicaciones en materia educativa, ya que facilita nuevas formas de aprendizaje y porque su dominio supone una buena preparación en relación con la vida adulta.

Es importante recalcar que estas tecnologías no solo son demandadas para favorecer aprendizajes y desarrollo de destrezas en el ámbito educativo, sino que también son una importante herramienta educativa en sí mismas.

El objetivo de introducir las tecnologías de la información en el proceso de enseñanzaaprendizaje no sólo mejora este proceso en diferentes asignaturas, sino que mejora la motivación de todos los agentes implicados, fomentando un entorno de trabajo más cooperativo y dinámico y favoreciendo el desarrollo de la autonomía en el proceso de aprendizaje del alumnado (posibilidad de monitorear su propio aprendizaje).

Por otra parte, el uso de estas tecnologías favorece el desarrollo de destrezas específicas (manejo de programas informáticos), y destrezas generales, siempre útiles en el desarrollo de la vida académica y profesional adulta (aquellas que tengan que ver con el análisis, la síntesis o la compilación de información.).

Pero hay que determinar hasta qué punto el uso de estas tecnologías favorece y/o potencia el aprendizaje en el ámbito escolar.

Desde este punto de vista, y como parte de una investigación realizada en 2003 por la OCDE desde su Programa Internacional de Evaluación Educativa, se ha realizado un estudio que compara diversos países ${ }^{102}$ y grupos sociales en orden a determinar la posible igualdad de acceso al ordenador de nuestros estudiantes adolescentes, y los diversos tipos de usos que le dan al ordenador. Se pretendía averiguar en qué medida estos jóvenes usan las nuevas tecnologías de la información, cuáles son sus actitudes hacia ellas, cuál es la relación entre el acceso al ordenador

101 Universidad de Oviedo.

102 Los países que han colaborado en la investigación son los que forman de la OCDE y otros como Túnez, Tailandia o Liechtenstein. Los países miembros de la OCDE son los siguientes: Australia, Austria, Bélgica, Canadá, República Checa, Dinamarca, Finlandia, Francia, Alemania, Grecia, Hungría, Islandia, Irlanda, Italia, Japón, Corea, Luxemburgo, México, Holanda, Nueva Zelanda, Noruega, Polonia, Portugal, República Eslovaca, España, Suiza, Suecia, Turquía, Gran Bretaña y Estados Unidos. 
y el rendimiento, y qué implicaciones se pueden desprender de este estudio para ser incorporadas en las políticas educativas y fomentar aprendizajes de calidad.

Ya en el año 2000, el Programa Internacional de Evaluación Educativa había realizado un estudio donde se determinaba un aumento significativo en cuanto al acceso al ordenador en casa y en la escuela. En ese momento se había concluido que los países participantes en el estudio (todos los que forman parte de la OCDE y otros que habían colaborado en el mismo, como Túnez o Tailandia) garantizaban un acceso casi universal al ordenador en ambos enclaves. El aumento respecto al acceso a las nuevas tecnologías por parte de los estudiantes ${ }^{103}$ fue especialmente alto en países donde previamente este acceso había sido comparativamente bajo, como Alemania, México o la República Checa.

Las principales conclusiones a las que ha llegado esta investigación son las siguientes:

Si hablamos de desigualdad respecto al acceso de los estudiantes al ordenador, hay que señalar que en un gran número de países muchos estudiantes no tienen acceso a las nuevas tecnologías, sobre todo en el hogar. Es el ejemplo de Grecia, Polonia, México o Turquía, y en general, de todos los países participantes en el estudio que no forman parte de la OCDE, excepto Liechtenstein.

Por otra parte, en la mayoría de los países, los estudiantes provenientes de los sectores menos privilegiados tienen significativamente menos probabilidades de acceder a ordenador en casa. La diferencia entre sectores sociales respecto al acceso a ordenador es significativa en países como México, Turquía o Tailandia. En países avanzados como Austria, Dinamarca o Islandia, las diferencias de acceso al ordenador en casa son pequeñas respecto de unos grupos sociales a otros. En este sentido, el 90\% de estudiantes provenientes de grupos desfavorecidos en países como Dinamarca, Suiza, Suecia o Islandia sí tienen acceso al ordenador en el hogar.

Aún así, incluso en países donde este acceso es más generalizado hay diferencias. En casi la mitad de los países de la OCDE, al menos un tercio de los estudiantes del grupo social menos favorecido no tienen ordenador en casa, y en países como Turquía, México, Polonia o Hungría la mayoría de los estudiantes pertenecientes a este grupo no tienen acceso al ordenador en sus hogares.

Es por ello fundamental garantizar, para todos los estudiantes, el acceso universal a las nuevas tecnologías en los centros educativos. En este sentido, apenas se encuentran diferencias de acceso al ordenador en la escuela según la procedencia social de los estudiantes, aunque en países como México, Túnez o Uruguay, el porcentaje diferencial de acceso entre los grupos más y menos privilegiados llega al $10 \%$.

Se puede concluir que en los países participantes en el estudio apenas se encuentran casos donde haya un problema generalizado de acceso a las nuevas tecnologías por parte de los estudiantes. Lo que sí encontramos son serias desventajas de acceso al ordenador en el hogar respecto de los estudiantes que provienen de grupos sociales más favorecidos en relación con los que provienen de los grupos más desfavorecidos.

Si hablamos del acceso a las nuevas tecnologías y su posterior utilización teniendo en cuenta el criterio de género, hay que señalar que la mayoría de estudiantes son capaces de

103 Los estudiantes participantes en el estudio tienen una media de 15 años de edad. Cabe destacar que si en los países de la OCDE la mayoría de las personas de esta edad están escolarizadas, en otros países como Tailandia esto no es así.

De aquí se desprende la necesidad de relativizar las conclusiones de este estudio, ya que únicamente han participado en el mismo las personas que en ese momento (año 2003) estaban escolarizadas. De esta manera, no se han tenido en cuenta a los adolescentes que han abandonado el sistema educativo a la hora de determinar la igualdad de acceso a las nuevas tecnologías por parte de los jóvenes. 
enfrentarse a las tareas básicas propias de las tecnologías de la información, y en general, confían en sí mismos respecto a sus habilidades cuando manejan Internet.

Sin embargo, aunque las chicas muestran la misma confianza que los chicos a la hora de realizar tareas básicas con el ordenador, son los chicos los que suelen desarrollar tareas de más alto nivel, como la programación o la creación de presentaciones multimedia, aventurándose en estas actividades incluso cuando son desconocidas para ellos. Esto sugiere que existen prejuicios respecto a los estudios que deben realizar chicos y chicas, siendo ellos los que en mayor número realizan estudios superiores relacionados con la informática.

La investigación concluye que algunos factores que permiten predecir ${ }^{104}$ las actitudes de los estudiantes ante las nuevas tecnologías de la información son: el género, la disponibilidad de ordenador en casa, con cuánta frecuencia lo usan y si han sido autodidactas en su uso.

Respecto al rendimiento académico de los estudiantes en relación con el uso que le dan al ordenador, es interesante recalcar que este uso se ve ampliamente afectado por los sentimientos que experimentan hacia el mismo, así como por el grado de confianza en sí mismos a la hora de desarrollar ciertas tareas con el ordenador.

Los estudiantes emplean las tecnologías de la información para una amplia gama de actividades, relacionadas con el entretenimiento, la educación o la comunicación. La mitad de los estudiantes participantes en el estudio juega frecuentemente a ordenador, y el mismo número lo usa para buscar información en Internet o para escribir textos en formato Word o similar.

Sólo una minoría emplea software específicamente educativo, aunque hay que resaltar que hay múltiples usos del ordenador que reportan beneficios educativos, como el empleo del correo electrónico o el uso de Internet para buscar información diversa.

$\mathrm{Y}$ aunque la asociación entre acceso y uso del ordenador y rendimiento no aporta evidencias causales directas del impacto del uso del ordenador en el aprendizaje, se puede señalar que aquellos estudiantes con acceso restringido al ordenador, que lo usan menos o que tienen menos confianza en sí mismos al utilizarlo obtienen un rendimiento académico menor.

Esto se debe en parte a que los estudiantes con menos posibilidad de acceso al ordenador provienen en muchos casos de grupos desfavorecidos. Aun así, el estatus socioeconómico no explica por entero la diferencia en el rendimiento entre estudiantes, tengan o no acceso a ordenador.

La cuestión es determinar hasta qué punto el uso de estas tecnologías en la escuela puede contribuir a crear una mayor igualdad en el rendimiento de todos los estudiantes.

De todas maneras, también hay que señalar que un uso muy elevado de las tecnologías de la información no implica necesariamente un mayor rendimiento por parte del alumnado. Los estudiantes que usan el ordenador con más frecuencia tienden a obtener, como media, un rendimiento menor que aquellos que usan el ordenador de forma más moderada. Esto implica que hay que analizar los distintos tipos de software que usan los alumnos.

En este sentido, hay que realizar estudios que determinen qué usos del ordenador son más efectivos y aumentan el rendimiento.

Así pues, en aquellos países donde el acceso a las nuevas tecnologías es prácticamente universal, las políticas propias del ámbito educativo deben asegurarse que su uso es efectivo, lo cual implica llevar a cabo programas de formación del profesorado y adecuar las nuevas tecnologías al horario y al currículum escolar.

104 Existen otros factores de predicción, como el status socio-económico de la familia a la que pertenece el alumno/a, la formación académica del padre y la madre, etc. 